\title{
A Customizable Multimodality Imaging Compound That Relates External Landmarks to Internal Structures
}

Mulugeta Semework

Department of Neuroscience, Columbia University, New York, New York

\begin{abstract}
Numerous research and clinical interventions, such as targeting drug deliveries or surgeries and finding blood clots, abscesses, or lesions, require accurate localization of various body parts. Individual differences in anatomy make it hard to use typical stereotactic procedures that rely on external landmarks and standardized atlases. For instance, it is not unusual to incorrectly place a craniotomy in brain surgery. This project was thus performed to find a new and easy method to correctly establish the relationship between external landmarks and medical scans of internal organs, such as specific regions of the brain. Methods: This paper introduces an MRI, CT, and radiographically visible compound that can be applied to any surface and therefore provide an external reference point to an internal (eye-invisible) structure. Results: Tested on nonhuman primates and isolated brain scans, this compound showed up with the same color in different scan types, making practical work possible. Conventional, and mostly of specific utility, products such as contrast agents were differentially colored or completely failed to show up and were not flexible. Conclusion: This compound can be customized to have different viscosities, colors, odors, and other characteristics. It can also be mixed with hardening materials such as acrylic for industrial or engineering uses, for example. Laparoscopy wands, electroencephalogram leads, and other equipment could also be embedded with or surrounded by the compound for ease in 3-dimensional visualizations. A pending U.S. patent endorses this invention.
\end{abstract}

Key Words: structural MRI; CT-scan; x-ray; imaging compound; neurosurgery

J Nucl Med Technol 2015; 43:267-274

DOI: 10.2967/jnmt.115.162404

$\mathbf{M}$ edical and engineering practices constantly employ devices and methods for visualizing internal structures in humans, animals, and machines. Three well-established imaging methods, MRI, CT, and radiography, are commonly used. Recently, integrated PET/MR systems have been drawing attention, and they are currently being used in the clinic to measure motion with high temporal resolution (1) and no additional instrumentation. The question still remains of

\footnotetext{
Received Jun. 17, 2015; revision accepted Aug. 11, 2015.

For correspondence or reprints contact: Mulugeta Semework, Columbia University, 1051 Riverside Dr., Unit 87, New York, NY 10032.

E-mail: msa2161@cumc.columbia.edu

Published online Sep. 3, 2015.

COPYRIGHT (C 2015 by the Society of Nuclear Medicine and Molecular Imaging, Inc.
}

how one can externally register an MRI, CT, or radiographically identified internal body part with those body parts visible to the naked eye. For instance, where should a surgeon make a precise $2-\mathrm{cm}$ skin incision to remove a bone outgrowth that is only $1 \mathrm{~cm}$ wide, if a $2-\mathrm{cm}$ incision is all the surgeon needs?

With its increased ease, power, and accessibility, especially the high resolution of T1-weighted structural images (2), MRI continues to be the medical imaging technique of choice. There has been growing interest in using this tool to study brain structure, function, development, and disease (3). However, unresolved technical and scientific difficulties, such as lack of means to scan and visualize certain objects, make it difficult to match interest with success. These problems are especially critical when one considers surgery on vital structures such as those in the brain-structures for which anatomy differs despite similarities in organization and relative location with respect to one another.

Individuals vary in their pattern of brain area folding, the shape and size of cortical areas, and relative locations (4). It is not uncommon for a craniotomy to be incorrectly placed when standardized atlases are used for localization of subcortical neural regions (5).

Because MRI is a noninvasive method that capitalizes on the complex mosaic across the cortical sheet (4), and because MRI permits visualization of the neural structure of the brain in vivo (5), it is possible to solve much of this problem by obtaining individual MR images before surgery, mapping structures of interest to an external marker, and then using the established coordinates during surgery.

The invention described in this paper aims at contributing to a solution by expanding on recent knowledge gained from preliminary research on nonhuman primates. It also builds on an old method of expressing relationships between surface markers: the use of skin markings to mathematically map internal structures of interest (6). Skin tattoos as outside markers were identified on MRI scans from vitamin $\mathrm{E}$ tablets affixed to the skin overlying the tattoos. This posed 3 major problems for wider use: first, vitamin E does not show up equally well and clearly on CT scans, radiographs, and several MRI sequences, such as T1-weighted, T2-weighted, and fluid-attenuated inversion recovery (FLAIR); second, because the tablets are not flexible and sizable and some body parts are not straight and accessible, the tablets cannot be affixed in all cases; and third, the tablets cannot stick to some body parts even though they are accessible and straight. 
FIGURE 1. Initial test of imaging compound (mix) and other ingredients on T1-weighted, T2-weighted, and T2-weighted FLAIR MRI sequences. Arrows indicate compound inside 2-mm-diameter surgical tubing. Commonly used vitamin $\mathrm{E}$ tablet disappears in T2-weighted sequences. Ingredient $1=$ olive oil; ingredient 2 = purified butter; future ingredient $3=$ water.

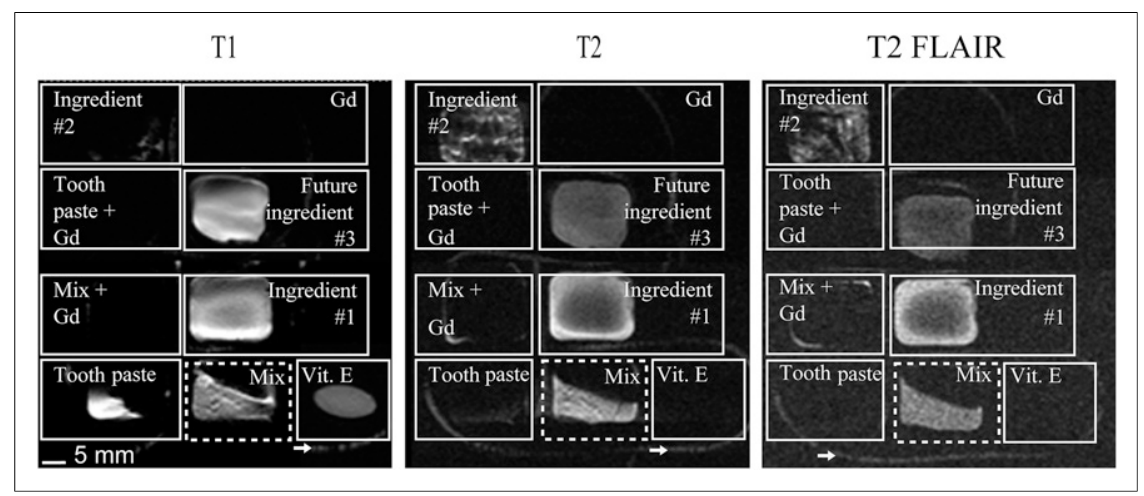

These problems call for a flexible, sizable, and safe substance that can be used in most MRI sequences and other scanning methods. This paper introduces such a substance, an imaging compound that is not yet commercially available.

\section{MATERIALS AND METHODS}

To replicate common problems, such as vitamin E and gadolinium showing up only on certain scans and under certain conditions, data from both the beginning and the ending stages of the development process are presented in this and the main Results sections.

In short, we tested the compound using monkey MRI scans and fixed monkey brain CT scans and radiographs and found it to have a well delineated radiodensity. It was not tested clinically. No human subjects were used, and there was therefore no need for Institutional Review Board approval. The Animal Care and Use Committees at
Columbia University and the New York State Psychiatric Institute approved all protocols as complying with the guidelines established in the Guide for the Care and Use of Laboratory Animals (7).

Figure 1 shows the initial testing results. The ingredients and the final mix were separately packed into different compartments of a small multipocketed plastic pouch. The compound was the only formulation that showed up more or less equally and clearly on T1-weighted, T2-weighted, and T2-weighted FLAIR MRI scans (Table 1 shows the parameters for all MRI sequences).

In general laboratory research practices, nonhuman primate skulls are routinely covered with dental acrylic (cement) either to protect a craniotomy or to secure a head-posting structure. Being an inert structure, the dental acrylic cannot be seen on any MRI scan. However, when the compound is used to cover the skull, the outlines of the compound can be visualized, allowing use in planning future

TABLE 1

MRI Parameters

\begin{tabular}{|c|c|c|c|c|c|c|c|c|c|c|}
\hline Figure & Series & Type & $\begin{array}{c}\text { Echo } \\
\text { time }(\mathrm{ms})\end{array}$ & $\begin{array}{l}\text { Repetition } \\
\text { time (ms) }\end{array}$ & $\begin{array}{l}\text { Inversion } \\
\text { time (ms) }\end{array}$ & $\begin{array}{l}\text { Thickness } \\
(\mathrm{mm})\end{array}$ & Matrix & Resolution & $\begin{array}{l}\text { Pixel spacing } \\
(\mathrm{mm})\end{array}$ & Scanner \\
\hline \multirow[t]{3}{*}{1} & T1 & $3 D$ & 4 & 34 & 0 & 1 & $120 \times 120$ & $256 \times 256$ & $0.47 \times 0.47$ & Signa \\
\hline & T2 & $2 \mathrm{D}$ & 97.27 & 5,200 & 0 & 2 & $120 \times 120$ & $256 \times 256$ & $0.47 \times 0.47$ & Signa \\
\hline & FLAIR & $2 \mathrm{D}$ & 120.64 & 10,002 & 2,200 & 2 & $120 \times 120$ & $224 \times 256$ & $0.47 \times 0.47$ & Signa \\
\hline \multirow[t]{4}{*}{2} & T2 fast spin echo & $2 \mathrm{D}$ & 105.76 & 10,818 & & 2 & $384 \times 256$ & $512 \times 512$ & $0.27 \times 0.27$ & Discovery \\
\hline & T1 FLAIR & $2 \mathrm{D}$ & 27.68 & $2,300.06$ & 970.54 & 2 & $320 \times 224$ & $512 \times 512$ & $0.27 \times 0.27$ & Discovery \\
\hline & T2 FLAIR & $2 \mathrm{D}$ & 125.60 & 8,000 & 2,250 & 2 & $320 \times 224$ & $512 \times 512$ & $0.27 \times 0.27$ & Discovery \\
\hline & BRAVO & $3 D$ & 4.10 & 9.36 & 450 & 2 & $384 \times 256$ & $512 \times 512$ & $0.27 \times 0.27$ & Discovery \\
\hline \multirow[t]{7}{*}{3} & T1 FLAIR & $2 \mathrm{D}$ & 16.81 & $3,000.01$ & 1,000 & 5 & $288 \times 192$ & $512 \times 512$ & $0.49 \times 0.49$ & Discovery \\
\hline & Diffusion-weighted & $2 \mathrm{D}$ & 80.8 & 8,000 & 0 & 5 & $128 \times 128$ & $224 \times 256$ & $0.94 \times 0.94$ & Discovery \\
\hline & T2 fast spin echo & $2 \mathrm{D}$ & 80.4 & 8,000 & 0 & 4 & $128 \times 128$ & $256 \times 256$ & $0.94 \times 0.94$ & Discovery \\
\hline & Diffusion tensor & $2 \mathrm{D}$ & 80.4 & 8,000 & 0 & 4 & $128 \times 128$ & $256 \times 256$ & $0.94 \times 0.94$ & Discovery \\
\hline & $\begin{array}{l}\text { Single-shot fast } \\
\text { spin echo }\end{array}$ & $2 \mathrm{D}$ & 40.10 & 953.78 & 0 & 5 & $288 \times 192$ & $512 \times 512$ & $0.43 \times 0.43$ & Discovery \\
\hline & SAG 3D FSPGR & $3 D$ & 2.55 & 6.32 & 500 & 1 & $128 \times 128$ & $256 \times 256$ & $0.98 \times 0.98$ & Discovery \\
\hline & $\begin{array}{l}\text { Fractional } \\
\text { anisotropy }\end{array}$ & $2 \mathrm{D}$ & 80.4 & 8,000 & 0 & 4 & $128 \times 128$ & $256 \times 256$ & $0.94 \times 0.94$ & Discovery \\
\hline \multirow[t]{5}{*}{4} & BRAVO & $3 D$ & 2.55 & 6.32 & 500 & 1 & $128 \times 128$ & $256 \times 256$ & $0.98 \times 0.98$ & Discovery \\
\hline & T1 FLAIR & $2 \mathrm{D}$ & 24 & 2,300 & & 2 & $320 \times 244$ & $140 \times 140$ & $0.44 \times 0.57$ & Discovery \\
\hline & T2 FLAIR & $2 \mathrm{D}$ & 120 & 8,000 & 2,250 & 2 & $320 \times 244$ & $140 \times 140$ & $0.44 \times 0.57$ & Discovery \\
\hline & MR angiography & $3 D$ & Out of phase & 23 & & 1.2 & $256 \times 192$ & $140 \times 105$ & $0.55 \times 0.55$ & Discovery \\
\hline & MR venography & $2 \mathrm{D}$ & Minimum & Minimum & & 1.5 & $256 \times 192$ & $140 \times 105$ & $0.55 \times 0.55$ & Discovery \\
\hline
\end{tabular}

*1.5-T Signa Excite (GE Healthcare).

†3-T Discovery MR750 (GE Healthcare).

SAG FSPGR = sagittal fast spoiled gradient-recalled echo. 


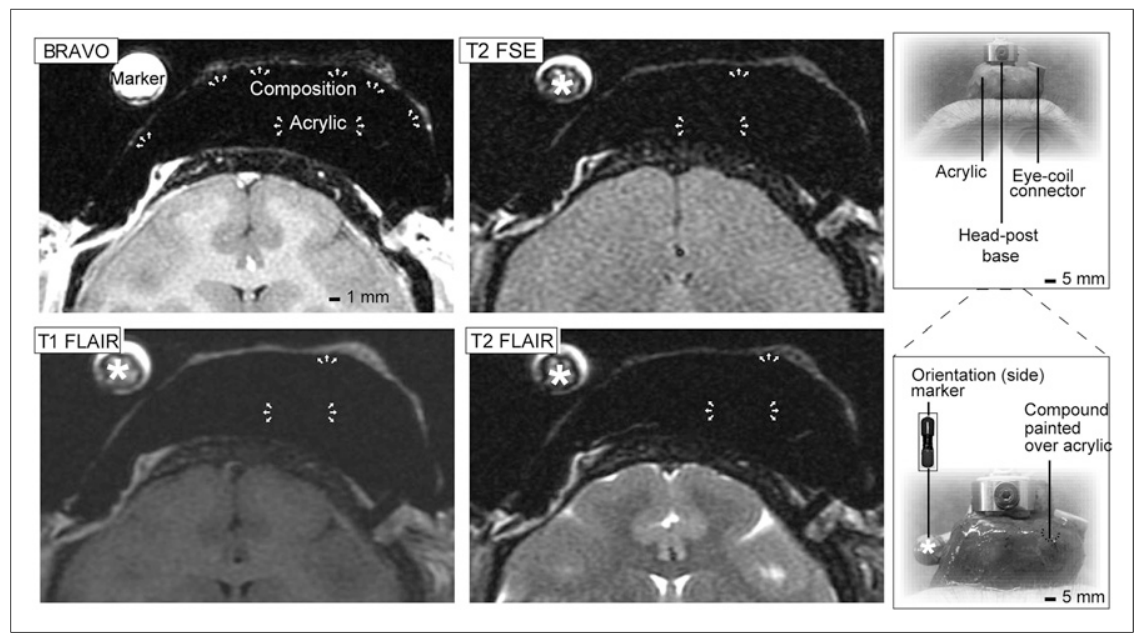

FIGURE 2. MRI test of compound in various monkey brain sequences. Thin, white layer of compound (top arrows) is seen on top of dental acrylic (opaque mound indicated by lower white arrows) in all scans. Photographs at right show dental acrylic implant in monkey, orientation marker (8-mm-diameter plastic tube filled with compound), and compound placement. FSE $=$ fast spin echo.

surgeries or following up any process and objects inside the dental acrylic or inside the brain (Fig. 2). By extension, the compound can be put over any body part, such as the skin over a patient's chest or neck, and the scan results can be used to plan interventions.

Because the greatest target is ultimately clinical utility, the final MRI testing of this product was performed in a setting that resembles common medical practice in two ways. First, MR scanning sequences were implemented with parameters used in patient evaluation procedures, such as T1 weighting, T2 weighting, fast spin echo, fractional anisotropy, single-shot fast spin echo, and 3-dimensional (3D) fast spoiled gradient echo. Second, several small vinyl tubes filled with the compound were glued to a plastic helmet (a swimming cap that mimics electroencephalogram caps, as shown in Fig. 3). This testing was done to demonstrate one possible use: a multipurpose wearable, imageable landmark overlying body parts (brain structures, for instance).

Shown in Figure 3 are the results from an assortment of scans of the compound in a network of surgical tubes glued to a plastic cap covering a phantom brain. The scans that do not show the compound clearly are those in which diffusion and anisotropy are imaged-scans that are not the most widely used in the clinic. However, the seemingly negative results in Figure 3 occurred mostly because the parameters for all scans had not been optimized at that stage. Further refinement (of the MR methods and the chemistry of the compound) resulted in more positive results (subsequent figures).

The constitution of the compound was modified for the second round of testing, which aimed at further ascertaining the preliminary results and including other MRI sequences and scan types (CT scans and radiographs).

The main ingredients of the 5 formulations of the compound and their proportions (by volume) were $30 \%-45 \%$ povidone-iodine or distilled water as the solvent and bright T2-weighted MR images (formulation 1), 15\%-20\% vitamin E oil (formulation 2), 5\%-10\% purified butter (formulation 3), 5\%-10\% slightly warmed olive oil (formulation 4), and other materials specific to project particulars ( $\leq 15 \%)$ (formulation 5 ).

The percentages of ingredients can be modified to create the desired consistency for various purposes. For instance, other ingredients (formulation 5) can be as high as $45 \%$. All the images presented here used $45 \%$ solvent. Users are encouraged to experiment with the numbers to fit their special needs. As commercial availability is anticipated to prove helpful in this regard, a U.S. patent is pending for the compound (part no. CU13187; Spectra Premium).

\section{RESULTS}

\section{MRI Scans}

It is important that any imaging substance encompass clinical MRI sequences whose use is expected to continue for the foreseeable future. The results for several clinical sequences indicated that the compound has great potential.
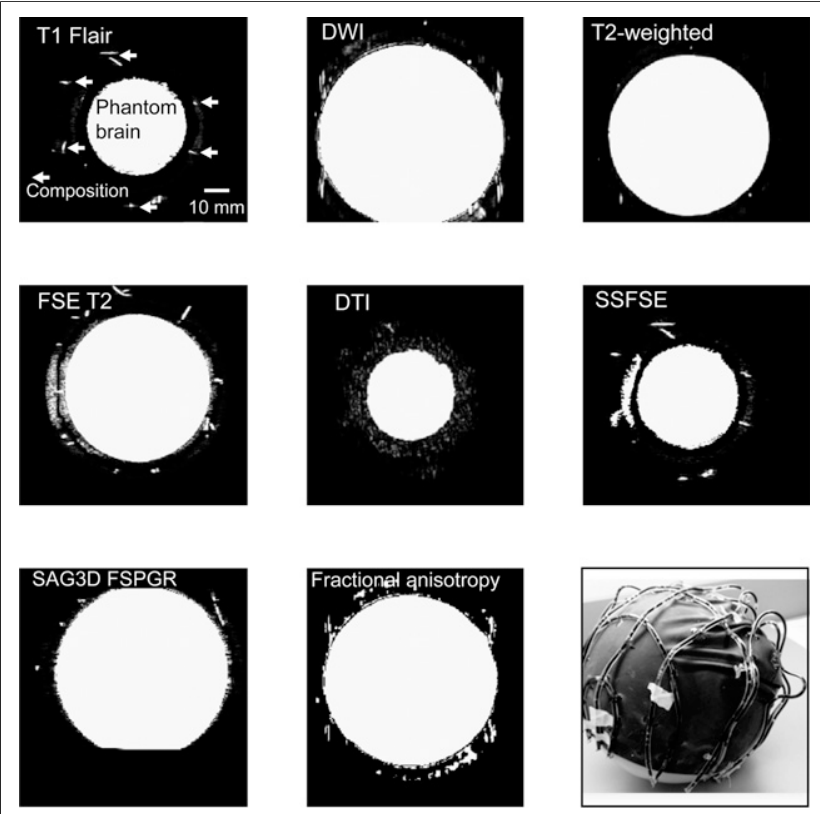

FIGURE 3. MRI test of compound in helmet-over-phantom scans. Arrows mark example locations in cross-sectional view of "brain," where compound is highly MR-visible. Almost all sequences show the compound. Photograph at bottom right shows phantom brain wearing plastic helmet with network of vinyl tubes containing compound. DTI = diffusion tensor imaging; DWI = diffusion-weighted imaging; FSE = fast spin echo; FSPGR = fast spoiled gradient-recalled echo; SAG3D = sagittal 3D; SSFSE = single-shot fast spin echo. 

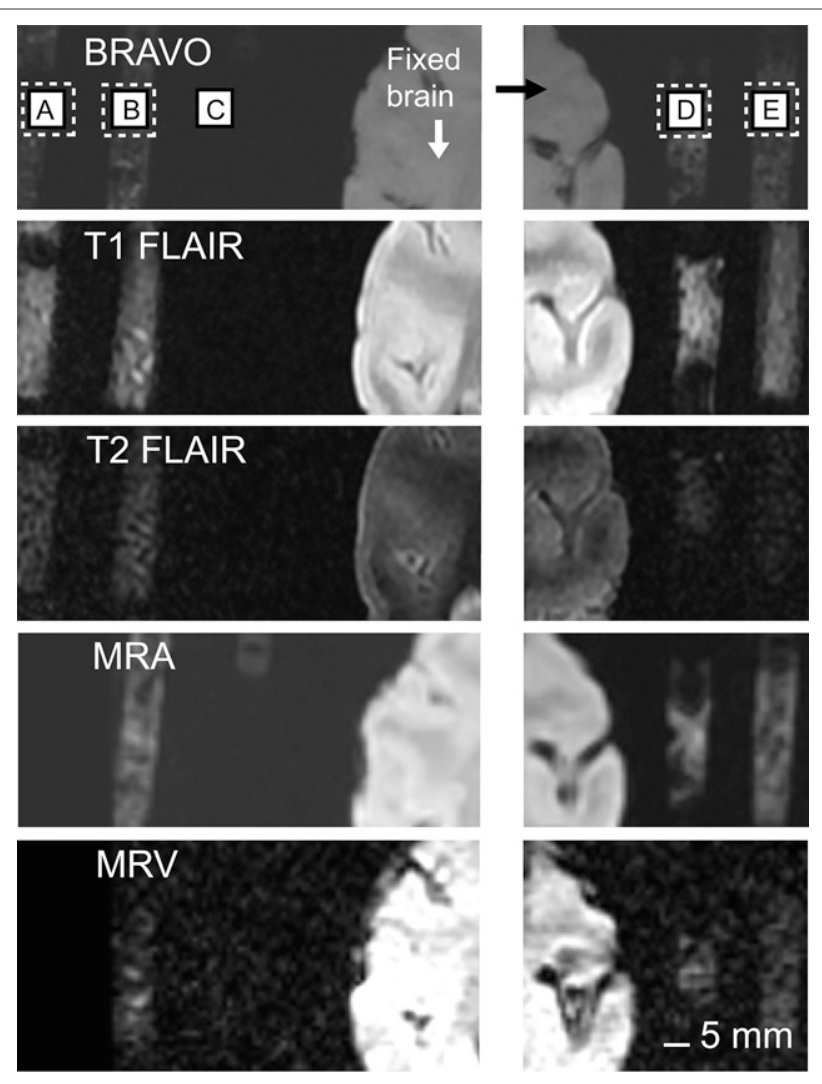

FIGURE 4. MRI test of various formulations of compound: formulation 1 mixed with dental acrylic $(A)$, formulation 1 mixed with acrylic (B), dental acrylic alone (C), formulation 1 without acrylic (D), and formulation 2 without acrylic (E). All were put in 1-mL surgical syringes for stability during scans and ease in visual comparisons. Dental acrylic alone is MRinvisible in all sequences. MRA = MR angiography; MRV = MR venography.

Depending on which pulse sequences were used, the compound was clearly visualized, having contrast and definition patterns similar to those of a T1-weighted brain MRI scan (Fig. 4). Once again, the main feature of the compound was that it consistently showed up as just one color (white) in all tested sequences. Color and brightness level for contrast agents, gray or white matter, and blood vessels varied.

\section{Radiographs}

The human body and other structures differentially attenuate $\mathrm{x}$-rays and create shadows that are captured by $\mathrm{x}$-ray-sensitive detectors. The transmitted fractions of $\mathrm{x}$-ray beams captured by the detectors are now widely used to study objects hidden to the naked eye. They have been especially useful in orthopedic and pulmonary medicine. However, because transmissions and attenuations depend on the opacity of imaged objects, it is still difficult to easily image the exact shape of objects that are relatively transparent, such as skin compared with bone. If one can use a chemical such as the compound discussed here to differentiate soft tissue, there will be no need for another kind of scan (MRI, for instance) to further analyze and understand the body part under study. For this reason, we acquired simultaneous radiographs of the compound and other structures, such as a fixed monkey brain and a stainless steel rod (Fig. 5; Table 2 provides the scan parameters). The scan results show that even under conditions of low-intensity image quality, the compound can be visualized. Understandably, the compound does not have the same intensity as stainless steel, but visibility is considerably improved.
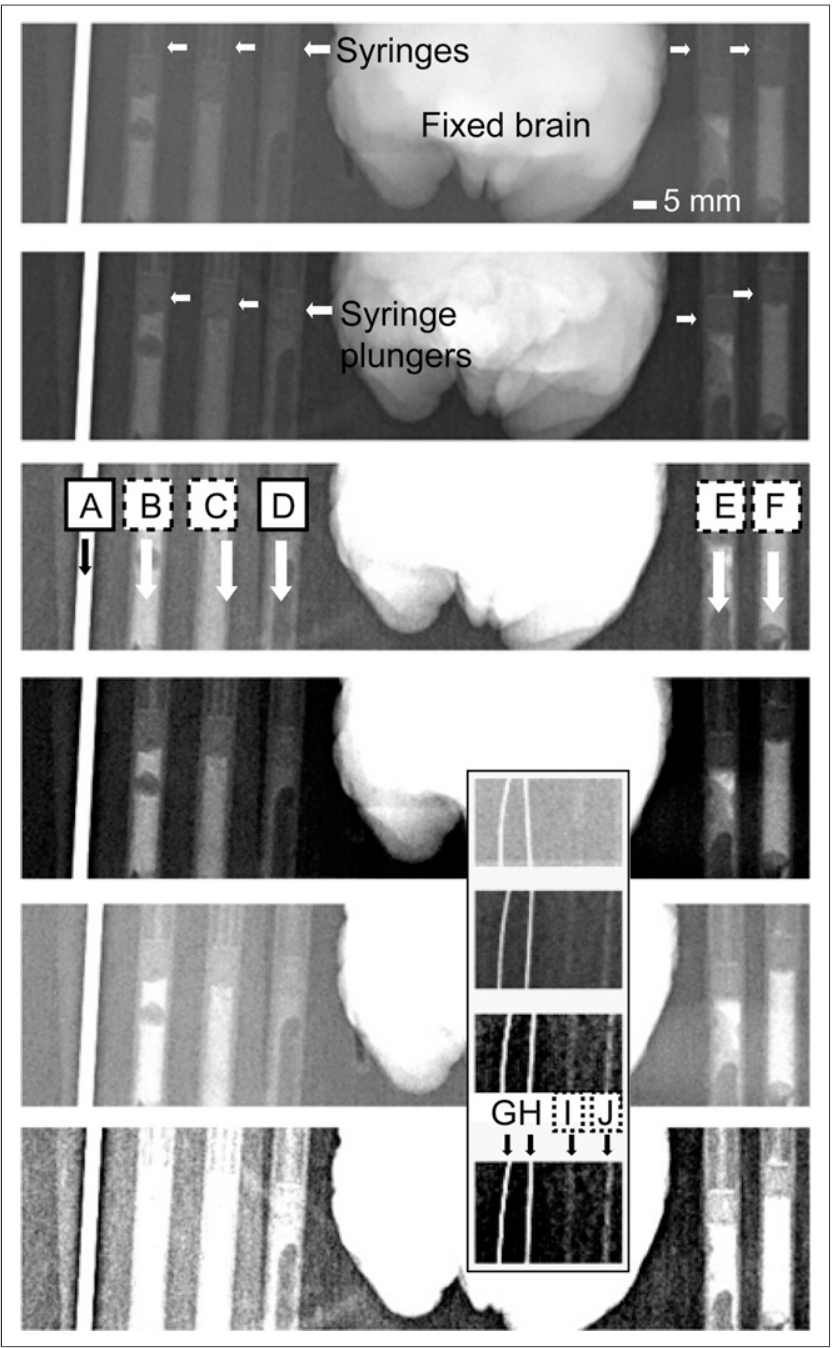

FIGURE 5. Radiography test of various formulations of compound: stainless steel guide tube (2-mm-diameter rod [A]), formulation 1 mixed with dental acrylic in 1-mL surgical syringe (B), formulation 2 mixed with acrylic in 1-mL surgical syringe (C), acrylic alone in 1-mL surgical syringe (D), formulation 1 without acrylic in 1-mL surgical syringe $(E)$, and formulation 2 without acrylic in 1-mL surgical syringe (F). Inset: $75-\mu \mathrm{m}$-thick microelectrode (G), $250-\mu \mathrm{m}$-thick microelectrode $(\mathrm{H})$, formulation 1 without acrylic in $0.5-\mathrm{mm}$-wide plastic guide tube (I), and formulation 2 without acrylic in $0.5-\mathrm{mm}$-wide plastic guide tube $(\mathrm{J})$. Same scan is presented with increasing contrast from top to bottom panels. 
TABLE 2

Radiography Parameters (Fig. 5)

\begin{tabular}{|c|c|c|c|c|c|c|c|c|c|}
\hline $\mathrm{kVp}$ & $\begin{array}{l}\text { Tube } \\
\text { current } \\
\text { (mA) }\end{array}$ & Modality & Filter & $\begin{array}{c}\text { Area-dose } \\
\text { product } \\
(\mathrm{dGy} \cdot \mathrm{cm} \cdot \mathrm{cm})\end{array}$ & Resolution & $\begin{array}{l}\text { Field } \\
\text { of view }\end{array}$ & $\begin{array}{c}\text { Exposure } \\
\text { (mAs) }\end{array}$ & $\begin{array}{c}\text { Pixel } \\
\text { spacing } \\
(\mathrm{mm})\end{array}$ & $\begin{array}{l}\text { Exposure } \\
\text { time (ms) }\end{array}$ \\
\hline 62 & 101 & $\begin{array}{l}\text { Bone } \\
\text { densitometry }\end{array}$ & $\begin{array}{l}\text { Multiple/ } \\
\text { copper }\end{array}$ & 0.09 & $865 \times 1,136$ & $173 \times 227$ & 1.61 & $0.19 \times 0.19$ & 16 \\
\hline
\end{tabular}

\section{CT Scans}

Following the success of radiography, which uses instruments that record the attenuation of x-ray beams as they pass through the imaged object, it was found possible to get crosssectional images by rotating the scanner $180^{\circ}$ around the body $(8,9)$. This method, called CT, has been widely used, but it shares the same shortcomings as MR and radiography. It too can benefit from a compound that can outline x-raytransparent objects. The images we captured in a CT scanner show that the compound is visible and has well-defined edges (Fig. 6; Table 3 provides the scan parameters).

Because there is a growing concern about radiation exposure and several approaches have been taken to reduce dose (9-11), the compound was scanned under both highand low-dose conditions (Fig. 7). The results show no critical and visible losses in image quality.

\section{DISCUSSION}

On MRI scans, CT scans, and radiographs, the tested compound was imageable when contained in tubes and when smeared over the acrylic head caps commonly used in monkeys to house and stabilize recording chambers and head-post sockets. The outline of the compound could be visualized on various scans, and its use in practical work was successful. Its overall performance points toward possible widespread use as a marker for various applications, whether for human and primate body parts or for research. For instance, a sleep or epilepsy research clinic may want to localize MRI-safe electroencephalogram leads on a patient to the underlying brain structures. MR images can be acquired after a dab of the compound is put on each lead, perhaps using larger amounts to mark suspected areas of epileptic foci, for example, and thus better associating structural images to experimental data. Conversely, the electrically conductive gel normally embedded in the connector terminals of electroencephalogram leads, electrocardiogram leads, and even laparoscopy and imaging wands can be manufactured using the compound, making 3D visualization possible without the need to continually apply compound to the surface.

It is anticipated that the compound will complement the use of internal contrast agents such as gadolinium, whose paramagnetic property has been extensively exploited despite being contraindicated in patients with renal dysfunction (12). Because it is highly unlikely that a single-element- based substance will be the external-surface equivalent of gadolinium, the compound is at the crossroads of great utility, price, and functionality. Although the compound may need to be perfected for some specialized MRI sequences, its testing has given encouraging results (Figs. 3 and 4).

T1- and T2-weighted sequences are used for visualization and quantification of fat and water, respectively (12-17). Both can be refined further using FLAIR pulse sequences to suppress fluid signals, especially cerebrospinal fluids in brain scanning (12), and are used to localize lesions and edematous tissue.
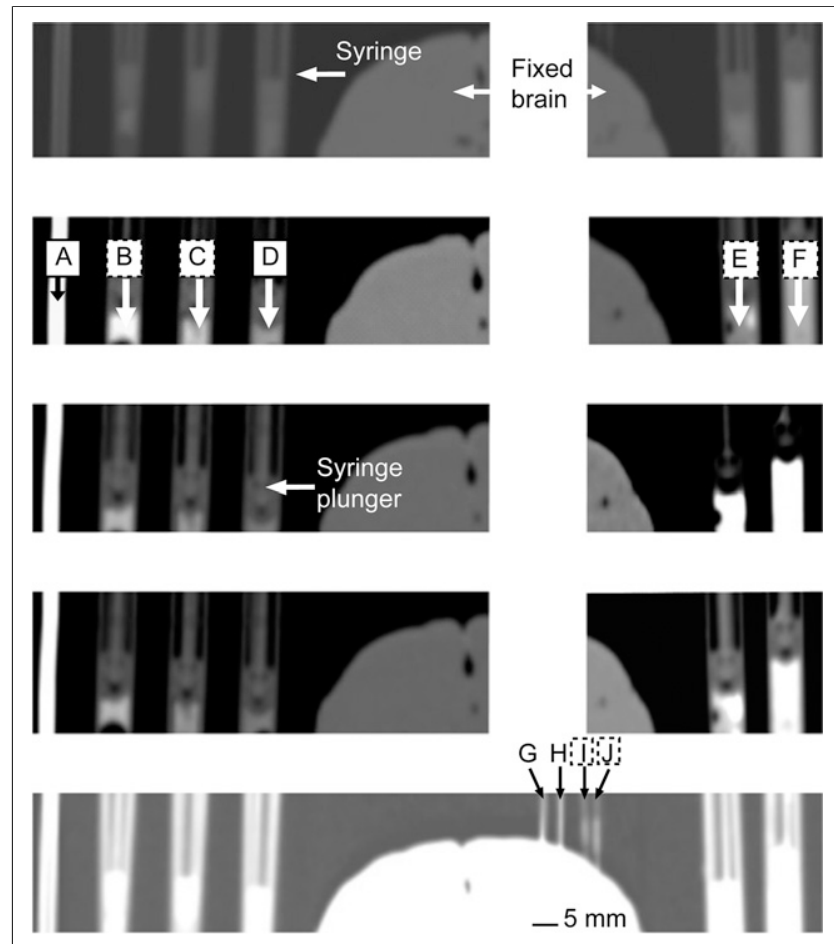

FIGURE 6. CT test of various formulations of compound: stainless steel guide tube (2-mm-diameter rod [A]), formulation 1 mixed with dental acrylic in 1-mL surgical syringe $(B)$, formulation 2 mixed with acrylic in 1-mL surgical syringe (C), acrylic alone in 1-mL surgical syringe (D), formulation 1 without acrylic in $1-\mathrm{mL}$ surgical syringe $(\mathrm{E})$, formulation 2 without acrylic in 1 -mL surgical syringe $(F), 75-\mu m$-thick microelectrode $(G)$, $250-\mu$ m-thick microelectrode $(H)$, formulation 1 without acrylic in 0.5-mm-wide plastic guide tube (I), and formulation 2 without acrylic in $0.5-\mathrm{mm}$-wide plastic guide tube $(\mathrm{J})$. Same scan is presented with increasing contrast from top to bottom panels. 
TABLE 3

CT Parameters

\begin{tabular}{|c|c|c|c|c|c|c|c|c|c|c|c|}
\hline $\begin{array}{l}\text { Scan } \\
\text { type }\end{array}$ & $\begin{array}{c}\text { Total } \\
\text { delivered } \\
(\mathrm{mAs})\end{array}$ & $\begin{array}{c}\text { Total } \\
\text { DLP } \\
(\mathrm{mGy} \cdot \mathrm{cm})\end{array}$ & Type & $\begin{array}{c}\text { Tube } \\
\text { potential } \\
(\mathrm{kV})\end{array}$ & $\mathrm{mAs}$ & $\begin{array}{c}\text { Reference } \\
\text { quality } \\
\text { milliamperage }\end{array}$ & $\begin{array}{c}\text { CT } \\
\text { dose index } \\
\text { volume } \\
\text { (mGy) }\end{array}$ & $\begin{array}{c}\text { DLP } \\
\mathrm{mGy} \cdot \mathrm{cm}\end{array}$ & $\begin{array}{c}\text { Rotation } \\
\text { time (s) }\end{array}$ & $\begin{array}{l}\text { Section } \\
\text { collimation } \\
(\mathrm{mm})\end{array}$ & Figure \\
\hline \multirow[t]{2}{*}{ High dose } & 1,628 & 190 & Topogram & 120 & 30 & $\mathrm{~mA}$ & & & 5.3 & 0.6 & $6 / 7$ \\
\hline & & & $\mathrm{HR}$ & & 120 & & 9.09 & 190 & 0.5 & & \\
\hline \multirow[t]{2}{*}{ Low dose } & 854 & 89 & Topogram & 120 & 35 & $\mathrm{~mA}$ & & & 5.3 & 0.62 & 7 \\
\hline & & & $\mathrm{HR}$ & & 50 & & 3.80 & 89 & 0.5 & & \\
\hline
\end{tabular}

MR angiography (artery imaging) and venography (vein imaging (18)) have clinical relevance and are routinely used. Abnormalities such as vein obstructions can also be identified by duplex ultrasound and CT scanning, in addition to MR venography (19). The sequences developed for oxygenated blood provide the best visualization in MR angiography.

Intraarterial catheter angiography - the other alternative for investigating serious complications such as intracranial vascular diseases - is slowly being replaced by angiography using CT or MRI because catheter angiography is invasive, requires that patients be admitted to the hospital, and has been associated with a $1 \%-3 \%$ rate of neurologic complications (20).

Another useful MR sequence is diffusion-weighted imaging. It is used in diagnosing pathologic conditions such as acute ischemia because it is highly sensitive to alterations in the molecular motion of water (21). The diffusion-weighted sequence has also been useful in discriminating various types of cancer, such as squamous cell carcinoma (22-24) and other tumors, from abscesses and autoimmune diseases such as Sjögren syndrome and Graves diseases (25).

The integrity of white matter is quantified and characterized by diffusion tensor imaging (26,27), which is based on the principle that, fundamentally, MRI is based on imaging of protons in moving water molecules. This movement is random (anisotropic) unless they are bound by scaffolds such as fibers and forced into axial or longitudinal diffusion (28). The diffusion tensor sequence thus allows one to study brain connectivity in addition to characterizing integrity. Accordingly, our compound can be incorporated into artificial fibers with a predetermined architecture based on the application. This use is in addition to and different from other applications of the compound, such as a material science application that uses the compound in artificial ceramic hips for car-crash simulations. The term artificial fibers specifically means viscous chemicals that have a locally nonrandom connectivity pattern, giving directionality to water molecule movement during MRI.
One common practice in guiding surgery is to register the head to MR images in reference to a $3 \mathrm{D}$ positioning sensor. Before surgery, T1-weighted MRI scans are acquired with fiducial markers fixed to the head (or, in animals, the head post). Such markers can be obtained from companies such as Brainsight (Rogue Research). During surgery the fiducial markers are reattached, and specific brain structures are registered and targeted using the MRI scan. Although this procedure is highly useful, it is possible to lose the marker signals in the initial scans either because the wrong MRI sequence is used or because the markers are too old. For instance, when we acquired BRAVO (inversion recovery-prepared, fast spoiled gradient echo sequence with parameters tuned to optimize brain tissue contrast; GE Healthcare (29)) and T1-weighted

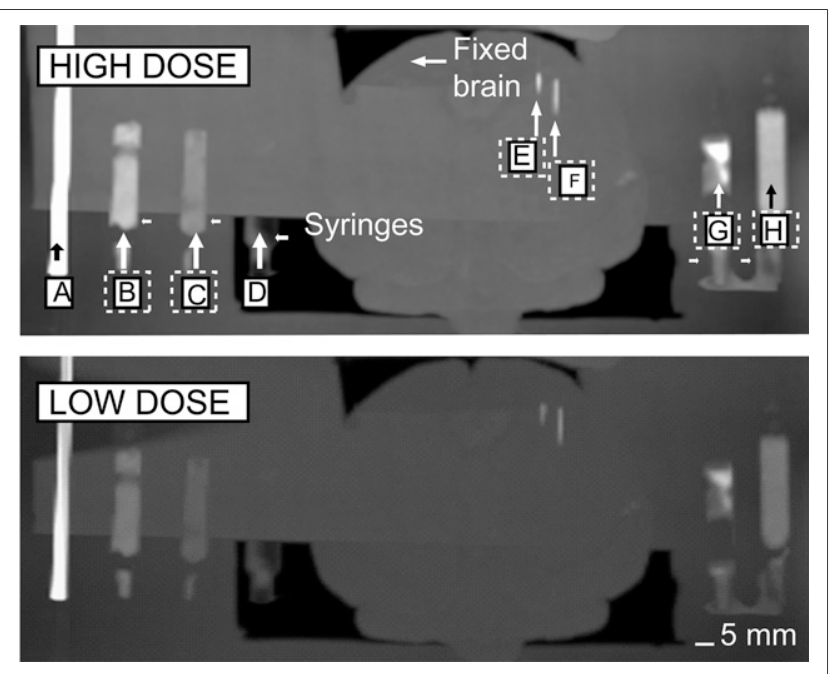

FIGURE 7. High- and low-dose 3D CT test of compound: stainless steel guide tube (2-mm-diameter rod $[A])$, formulation 1 mixed with dental acrylic in 1-mL surgical syringe (B), formulation 2 mixed with acrylic in 1-mL surgical syringe (C), acrylic alone in 1-mL surgical syringe (D), 75- $\mu$ m-thick microelectrode $(E), 250-\mu$ m-thick microelectrode $(F)$, formulation 1 without acrylic in $0.5-\mathrm{mm}$-wide plastic guide tube $(\mathrm{G})$, and formulation 2 without acrylic in 0.5-mm-wide plastic guide tube $(H)$. 
scans using the Brainsight marker, we could not see the markers until we painted them with our compound. Moreover, unlike our compound, whose flexibility can give a stable reference by providing a perfect contour of the surface to which it is affixed, the rigid fiducial markers may cause errors. Chen et al. (30) have discussed some of the potential complications in detail. They indicated that errors may arise if the markers are not firmly attached to the imaged subject, if pressure is applied to the markers during imaging, and if the position of markers changes between imaging and surgery, as can happen during patient transportation, surgical prepping, and head-clamp attachment. Chen et al. also indicated other possible limitations, such as the increase in targeting error that occurs as depth increases, based on the extrapolation distance from the markers. The problem of preventing pressure from being applied to the markers is sometimes difficult to solve; for instance, in our primate scans we use a custommade coil that goes inside the stereotactic apparatus and over the head.

Because the compound is made of more-or-less natural products, we anticipate that it can be manufactured as a sterile product that can easily and safely be applied to exposed body parts, including the dura of the brain. The compound can be customized to have different viscosities, colors, odors, and other characteristics. Further testing and improvement of the compound and marketing negotiations are currently under way.

One encouraging recent development-the availability of hybrid PET/MR systems $(1,31)$ - is an ideal setting for the compound. The integration of anatomic, functional, and molecular imaging, as well as of biologic data with medical imaging, is essential and is becoming increasingly possible (32). Such technologic advances call for matching advances in auxiliary equipment such as this compound, which helps register highresolution internal anatomic images with external markers.

\section{CONCLUSION}

The great advantage of the compound introduced here is that it has well-defined boundaries without evident artifacts, is physically flexible, and is the same color on different types of scans whereas most other options such as contrast agents and vitamin E tablets are differentially colored on scans or are not seen at all. Future evaluations of this compound need to be completed with great care and with an understanding of the possible interference artifacts and stability variations, particularly those that could arise from specialized formulations.

\section{DISCLOSURE}

This work was supported by a NINDS training grant (2T32MH015174-35) to Dr. Hen, an NEI grant (1 R01 EY014978-06) to Dr. Goldberg, a supplement grant through the NEI (3 R01 EY014978-06), and the Brain \& Behavior Research Foundation (NARSAD) 2013 Young Investigator
Award. Dr. Semework and Columbia University have a patent application pending for the compound. No other author has a financial or proprietary interest in the material or methods mentioned. There is no other potential conflict of interest relevant to this article.

\section{ACKNOWLEDGMENTS}

Heartfelt gratitude is given to Dr. Michael E. Goldberg for his tireless support of this project. Generous scan time and technical and expert support were provided by Andrew Gerber, director of the MRI Unit at New York State Psychiatric Institute, and his staff; Chaitanya R. Divgi, director of the PET Center at Columbia University Medical Center Department of Radiology, and his staff; Stephen Dashnaw, imaging supervisor of the Center for Neurobiology and Behavior at the Columbia University/Neurological Institute Department of Radiology, and his staff; Roy Thompson, radiology manager at New York-Presbyterian Hospital; and Ron Katz and his colleagues at Columbia Technology Ventures.

\section{REFERENCES}

1. Disselhorst JA, Bezrukov I, Kolb A, Parl C, Pichler BJ. Principles of PET/MR imaging. J Nucl Med. 2014;55(suppl 2):2S-10S.

2. Saad ZS, Glen DR, Chen G, Beauchamp MS, Desai R, Cox RW. A new method for improving functional-to-structural MRI alignment using local Pearson correlation. Neuroimage. 2009;44:839-848.

3. Smith SM, Jenkinson M, Woolrich MW, et al. Advances in functional and structural MR image analysis and implementation as FSL. Neuroimage. 2004;23 (suppl 1):S208-S219.

4. Van Essen DC, Drury HA, Joshi S, Miller MI. Functional and structural mapping of human cerebral cortex: solutions are in the surfaces. Proc Natl Acad Sci USA. 1998;95:788-795.

5. Saunders RC, Aigner TG, Frank JA. Magnetic resonance imaging of the rhesus monkey brain: use for stereotactic neurosurgery. Exp Brain Res. 1990;81:443446.

6. Semework M. A simple structural magnetic resonance imaging (MRI) method for 3D mapping between head skin tattoos and brain landmarks. Presented at: Southern Biomedical Engineers Conference; 2010; College Park, MD.

7. Guide for the Care and Use of Laboratory Animals. 8th ed. Washington, DC: National Academy Press; 2011.

8. Robb RA. X-ray computed tomography: an engineering synthesis of multiscientific principles. Crit Rev Biomed Eng. 1982;7:265-333.

9. Trattner S, Pearson GD, Chin C, et al. Standardization and optimization of CT protocols to achieve low dose. J Am Coll Radiol. 2014;11:271-278.

10. Dougeni E, Faulkner K, Panayiotakis G. A review of patient dose and optimisation methods in adult and paediatric CT scanning. Eur J Radiol. 2012;81: e665-e683.

11. Prokop M. Radiation dose in computed tomography. Risks and challenges [in German]. Radiologe. 2008;48:229-242.

12. Biglands JD, Radjenovic A, Ridgway JP. Cardiovascular magnetic resonance physics for clinicians: part II. J Cardiovasc Magn Reson. 2012;14:66.

13. $\mathrm{Hu} \mathrm{HH}$, Kan HE. Quantitative proton MR techniques for measuring fat. NMR Biomed. 2013;26:1609-1629.

14. Goodpaster BH. Measuring body fat distribution and content in humans. Curr Opin Clin Nutr Metab Care. 2002;5:481-487.

15. McMahon KL, Cowin G, Galloway G. Magnetic resonance imaging: the underlying principles. J Orthop Sports Phys Ther. 2011;41:806-819.

16. Panigrahy A, Borzage M, Bluml S. Basic principles and concepts underlying recent advances in magnetic resonance imaging of the developing brain. Semin Perinatol. 2010;34:3-19.

17. Ridgway JP. Cardiovascular magnetic resonance physics for clinicians: part I. J Cardiovasc Magn Reson. 2010;12:71. 
18. Spritzer CE. Progress in MR imaging of the venous system. Perspect Vasc Surg Endovasc Ther. 2009;21:105-116.

19. Arnoldussen CW, de Graaf R, Wittens $\mathrm{CH}$, de Haan MW. Value of magnetic resonance venography and computed tomographic venography in lower extremity chronic venous disease. Phlebology. 2013;28(suppl 1):169-175.

20. Jäger HR, Grieve JP. Advances in non-invasive imaging of intracranial vascular disease. Ann R Coll Surg Engl. 2000;82:1-5.

21. Schafer J, Srinivasan A, Mukherji S. Diffusion magnetic resonance imaging in the head and neck. Magn Reson Imaging Clin N Am. 2011;19:55-67.

22. Vandecaveye V, De Keyzer F, Dirix P, Lambrecht M, Nuyts S, Hermans R. Applications of diffusion-weighted magnetic resonance imaging in head and neck squamous cell carcinoma. Neuroradiology. 2010;52:773-784.

23. Friedrich KM, Matzek W, Gentzsch S, Sulzbacher I, Czerny C, Herneth AM. Diffusion-weighted magnetic resonance imaging of head and neck squamous cell carcinomas. Eur J Radiol. 2008;68:493-498.

24. Kim S, Loevner L, Quon H, et al. Diffusion-weighted magnetic resonance imaging for predicting and detecting early response to chemoradiation therapy of squamous cell carcinomas of the head and neck. Clin Cancer Res. 2009;15:986994.

25. Razek AA. Diffusion-weighted magnetic resonance imaging of head and neck. J Comput Assist Tomogr. 2010;34:808-815.
26. Chanraud S, Zahr N, Sullivan EV, Pfefferbaum A. MR diffusion tensor imaging: a window into white matter integrity of the working brain. Neuropsychol Rev. 2010;20:209-225.

27. Viallon M, Cuvinciuc V, Delattre B, et al. State-of-the-art MRI techniques in neuroradiology: principles, pitfalls, and clinical applications. Neuroradiology. 2015;57:441-467.

28. Song SK, Sun SW, Ramsbottom MJ, Chang C, Russell J, Cross AH. Dysmyelination revealed through MRI as increased radial (but unchanged axial) diffusion of water. Neuroimage. 2002;17:1429-1436.

29. BRAVO. GE Healthcare website. http://www3.gehealthcare.com/en/products/ categories/magnetic_resonance_imaging/neuro_imaging/bravo. Updated June 20, 2012; Accessed September 21, 2015.

30. Chen AV, Wininger FA, Frey S, et al. Description and validation of a magnetic resonance imaging-guided stereotactic brain biopsy device in the dog. Vet Radiol Ultrasound. 2012;53:150-156.

31. Runge VM. Current technological advances in magnetic resonance with critical impact for clinical diagnosis and therapy. Invest Radiol. 2013;48: 869-877.

32. Huang HM, Shih YY. Pushing CT and MR imaging to the molecular level for studying the "omics": current challenges and advancements. Biomed Res Int. 2014;2014:365812. 